\title{
Grands projets : Trop grands pour réussir?
}

\author{
Lavagnon A. Ika ${ }^{1}$
}

« Le chemin est long du projet à la chose »-Molière

\section{INTRODUCTION}

\section{Le phénomène du « too big to fail » au} niveau des projets se dédouble d'un paradoxe : malgré des dépassements de coût élevés et récurrents, les grands projets prolifèrent et leur économie semble prospérer. On les chiffre à plus de 40000 milliards \$.

« Vous êtes un chef de projet d'expérience. Votre impressionnant parcours professionnel est jalonné de grands projets. Bien sûr, les dépassements de délai et de coût vous sont familiers. De grands succès. De grands échecs aussi. Fort de cette longue et riche expérience, vous jouez, à l'automne de votre carrière, un rôle de premier plan dans le coaching et la formation des jeunes chefs de projet dans votre organisation. Dans le cadre d'un atelier sur la réalisation des grands projets, l'un d'eux vous demande: les grands projets sont-ils trop grands pour réussir?»

La maxime «Big is beautiful» que l'on pourrait traduire par «ce qui est efficace doit être nécessairement grand » n'est ni nouvelle ni propre aux projets. Il y a plus d'un siècle, le juriste Louis Brandeis fustigeait la grande taille de certaines compagnies américaines et avertissait contre ce qu'il appelait alors «the curse of bigness » ou «la malédiction de la grande taille ». Plus près de nous encore, en économie du développement, il y a exactement 40 ans, Ernst Friedrich Schumaker, dénonçait, lui aussi, dans son livre à succès «Small is beautiful », les ratés du «Big is beautiful » et incitait fortement à embrasser plutôt les vertus de la petite taille pour réussir. Comme Louis Brandeis avant lui, Schumaker n'a pas fait l'unanimité et a été critiqué, notamment, pour ce qui concerne la «bonne taille» des projets de développement international.

Mais la récente crise financière de 2008 a redonné du lustre au «Small is beautiful » et il y a, depuis, un regain d'intérêt pour deux de ses chantres les plus célèbres. Tout le monde connaît désormais la formule «too big to fail» ou «trop grand pour échouer» qui a fait couler beaucoup d'encre et de salive dans les cercles économiques et financiers. De façon un peu simpliste, elle consacre le phénomène suivant : Certaines banques, notamment américaines, sont devenues tellement grosses qu'il en coûtera moins cher au gouvernement de les sauver que de les laisser faire défaut. Le même phénomène s'observe au niveau des grands projets. Ces derniers semblent, en règle générale, trop grands pour échouer: on sous-estime (systématiquement?) leurs coûts et, au milieu de leur réalisation, on fait valoir qu'il est tard, voire même trop tard d'y renoncer, compte tenu de leurs nombreux avantages et des coûts irrécupérables, mais importants jusque-là investis. Ainsi, on se retrouve fréquemment avec des coûts finaux de réalisation exorbitants.

Le phénomène du «too big to fail » au niveau des projets se dédouble d'un paradoxe : malgré des dépassements de coût élevés et récurrents, les grands projets prolifèrent et leur économie semble prospérer ${ }^{2}$. On les chiffre à plus de 40000 milliards \$. Cependant, plus d'un projet 
sur deux ne livre pas l'impact attendu. Que ces statistiques soient valides ou pas n'est pas le problème crucial ${ }^{3}$. Plus important encore est le fait que les projets échouent souvent voire très souvent. Mais on peut se demander si les grands projets réussissent mieux que les petits projets ou si la malédiction de la taille (ou de l'envergure) frappe les grands projets ou encore, plus concrètement, si le budget des grands projets est un enjeu crucial pour leur succès ou échec. En conséquence, cet article aborde la question: Les grands projets sont-ils trop grands pour réussir « too big to succeed »?

Dans ce texte, nous mettons en évidence la performance des grands projets et nous y soulignons spécifiquement le lien entre leur budget et leur performance. Premièrement et à l'aide d'exemples concrets dans le monde, au Canada et au Québec, nous ferons un bref retour sur ce que nous savons déjà sur la performance des projets et le lien avec leur taille, et plus précisément, leur budget. À l'instar de Flyvbjerg ${ }^{4}$, nous considérons qu'un grand projet, par définition, coûte au total, pour sa réalisation, entre des centaines de millions et plusieurs milliards de dollars. Deuxièmement, nous aborderons brièvement les problèmes que posent les grands projets en mettant l'accent sur l'exemple du Rapibus de Gatineau. Troisièmement et finalement, nous terminerons par des réflexions sur la question qui est au cœur de cet article: les grands projets sont-ils trop grands pour réussir?

\section{QUE SAVONS-NOUS DE LA PERFORMANCE DES GRANDS PROJETS?}

La performance des grands projets défraie souvent la chronique. On peut penser aux Jeux Olympiques d'hiver de Sotchi 2014 dont le budget a été estimé à environ 10 milliards \$ et qui auront coûté plus de 50 milliards \$. Les grands projets, quand ils ne sont pas abandonnés, sont souvent en dépassement de délai et de coût.

La performance des grands projets défraie souvent la chronique. On peut penser aux Jeux Olympiques d'hiver de Sotchi 2014 dont le budget a été estimé à environ 10 milliards $\$$ et qui auront coûté plus de 50 milliards \$. Les grands projets, quand ils ne sont pas abandonnés, sont souvent en dépassement de délai et de coût. Mais, que nous apprend la recherche sur les dépassements de coût des grands projets? Le professeur Alfred Hirschman ${ }^{5}$ économiste de développement et pionnier malheureusement méconnu de la gestion de projet - est probablement l'un des tout premiers auteurs à avoir étudié les grands projets. Après avoir analysé 11 grands projets de la Banque mondiale en Europe, en Amérique latine, en Asie et en Afrique, Hirschman notait déjà des dépassements de coût certains mais qu'il n'a curieusement pas chiffrés. Certes, on peut penser que les projets de développement international connaissent des dépassements de coût en raison de leur complexité sociopolitique élevée et du contexte singulier dans lequel ils sont réalisés. Mais le dépassement de coût n'est pas un phénomène qui leur est propre. Les travaux de recherche en attestent. Pêle-mêle, les chiffres suivants témoignent d'un dépassement de coût notable, récurrent et généralisé à presque tous les types de projet (voir le Tableau 1 qui suit).

\section{Une étude de 52 grands projets civils de 500 millions $\$$ à plus de 10 milliards \$ révèle un dépassement de coût moyen estimé à 2 millions \$.}

Une étude de 52 grands projets civils de 500 millions $\$$ à plus de 10 milliards $\$$ révèle un dépassement de coût moyen estimé à 2 millions $\$ 6$. Dans leur analyse de 34 études couvrant 1536 projets réalisés dans les années 1960 et 1970 et huit grands projets dont le Concorde, Morris et $\operatorname{Hough}^{7}$ avancent un dépassement de coût moyen de l'ordre de $40 \%$ à $200 \%$ du budget imparti pour un projet de construction sur deux. 
Tableau 1 - La performance des grands projets : des résultats de recherche et des statistiques sur les dépassements de coût

\begin{tabular}{|c|c|c|c|}
\hline Auteurs & Type de projet & $\begin{array}{l}\text { Envergure de } \\
\text { l'étude }\end{array}$ & $\begin{array}{l}\text { Dépassement de coût } \\
\text { moyen }\end{array}$ \\
\hline $\begin{array}{l}\text { Merrow et al. } \\
(1988)\end{array}$ & Projets civils & $\begin{array}{c}52 \text { grands projets de } \\
500 \text { millions } \$ \text { à } 10 \\
\text { milliards } \$\end{array}$ & 2 millions \$ \\
\hline $\begin{array}{l}\text { Morris et Hough } \\
\text { (1987) }\end{array}$ & $\begin{array}{c}\text { Divers } \\
\text { (construction, } \\
\text { aéronautique, etc.) }\end{array}$ & $\begin{array}{l}34 \text { études couvrant } \\
1536 \text { projets plus } 8 \\
\text { grands projets dont } \\
\text { Concorde et Fulmar. }\end{array}$ & $40 \%$ à $200 \%$ \\
\hline $\begin{array}{l}\text { Miller et Lessard } \\
(2000)\end{array}$ & Ingénierie & 60 projets & 1 projet sur 5 \\
\hline Meier (2008) & Acquisition militaire & 26 projets USA & $15 \%$ \\
\hline Flyvbjerg (2005) & $\begin{array}{l}\text { Transport (Routes, } \\
\text { tunnels, ponts, } \\
\text { chemins de fer) }\end{array}$ & $\begin{array}{l}258 \text { projets d'une } \\
\text { valeur totale de } 90 \\
\text { milliards } \$ \text { sur } 5 \\
\text { continents }\end{array}$ & $\begin{array}{l}9 \text { projets sur } 10 ; 20 \% \\
\text { pour chemins de fer; } \\
34 \% \text { ponts et tunnels et } \\
20 \% \text { pour routes }\end{array}$ \\
\hline $\begin{array}{l}\text { Hertogh et al. } \\
(2008)\end{array}$ & Infrastructure & $\begin{array}{l}15 \text { projets de la } \\
\text { Commission } \\
\text { européenne }\end{array}$ & Tous les 15 projets \\
\hline Omega (2012) & Transport & $\begin{array}{c}30 \text { grands projets } \\
\text { (ex. : Big Dig de } \\
\text { Boston, TGV } \\
\text { Valence-Marseille) }\end{array}$ & $22 \%$ \\
\hline $\begin{array}{l}\text { Flyvbjerg et } \\
\text { Budzier (2011) }\end{array}$ & $\begin{array}{l}\text { Technologies de } \\
\text { l'information (TI) }\end{array}$ & $\begin{array}{c}1471 \text { projets; coût } \\
\text { moyen de } 167 \\
\text { millions; plus cher : } \\
32 \text { milliards } \$\end{array}$ & $27 \%$ \\
\hline $\begin{array}{l}\text { Jergeas et } \\
\text { Ruwanpura } \\
\text { (2010) }\end{array}$ & $\begin{array}{c}\text { Projets pétroliers et } \\
\text { gaziers }\end{array}$ & $\begin{array}{l}\text { Sables bitumineux } \\
\text { de l'Alberta }\end{array}$ & $50 \%$ à $100 \%$ \\
\hline
\end{tabular}

Mais qu'en est-il des projets d'ingénierie? Un projet d'ingénierie sur cinq subit un dépassement de coût ${ }^{8}$. Les projets d'acquisition militaire ne font pas non plus exception. Une étude de 26 projets d'acquisition militaire avance un dépassement moyen de coût de $15 \%{ }^{9}$.

Des travaux menés sur les projets de transport confortent cette tendance au dépassement de coût. L'étude probablement la plus marquante en la matière revient à Flyvbjerg ${ }^{10}$. Après avoir examiné plus de 258 grands projets de transport (routes, tunnels, ponts, chemins de fer) d'une valeur totale de 90 milliards, il conclut que: neuf sur 10 dépassent le budget; que les dépassements de coût ont eu lieu dans 20 pays et sur cinq continents et qu'ils ont été récurrents sur une période aussi longue que 70 ans. Également, sur les 15 projets d'infrastructure tels que les chemins de fer et les routes qui ont fait l'objet d'étude dans le cadre du programme Netlipse de la Commission européenne, aucun n'a respecté le budget imparti ${ }^{11}$. De plus, il ressort d'une analyse des coûts de 30 grands 
projets de transport dont le Big Dig de Boston, le métro $d$ 'Athènes et le train à grande vitesse (TGV) Valence -Marseille que le dépassement de coût moyen est de $22 \%$ du budget ${ }^{12}$.

Le phénomène du dépassement de coût s'étend aussi aux projets de TI. Le Groupe Standish a analysé une base de plus de 50000 projets de TI petits ou grands - et constaté que les dépassements oscillent autour de $50 \%$ de 2004 à 2012 et surtout que les grands projets n'ont virtuellement aucune chance de rentrer dans le budget ${ }^{13}$. Une étude de 1471 projets de TI réalisée par Flyvbjerg et Budzier $^{14}$ en 2011 montre que le dépassement de coût moyen est de $27 \%$ et qu'un projet sur six connaît un dépassement de plus de $200 \%$ en moyenne. Même, plus près de nous, les projets pétroliers et gaziers des sables bitumineux de l'Alberta affichent des dépassements de coût de $50 \%$ à $100 \%{ }^{15}$.

Mais quel est le degré de précision de ces chiffres de dépassement de coût? Il varie, en effet, selon que le budget approuvé pour le projet - la base de l'estimation - a été fixé avant ou après les études de faisabilité ou bien la planification du projet. Bien sûr, avec un budget estimé après la planification, puis judicieusement mis à jour et enfin établi juste avant la réalisation du projet, il est raisonnable de s'attendre à un dépassement de coût moindre. Par ailleurs, il est normal qu'un projet connaisse un dépassement de coût plus ou moins important si le client fait des changements à son envergure en cours de réalisation. Pour ces raisons, on pourrait s'objecter à la validité de ces statistiques de dépassement de coût et accepter comme Benjamin Disraeli que : «Il y a trois sortes de mensonges: les mensonges, les foutus mensonges et les statistiques ». Trêves de statistiques sur les dépassements de coût des grands projets! Que dire de la performance de quelques grands projets dans le monde, au Canada et au Québec? Le tableau 2 ci-après en donne une illustration sommaire et montre, en somme, que le phénomène du dépassement des coûts des grands projets ne date pas d'aujourd'hui; il est bien réel, récurrent et généralisé dans presque tous les secteurs d'activité. Mais qu'est-ce qui peut expliquer cette performance de coût décevante ou de quels grands maux souffrent les grands projets?

Tableau 2 - Les dépassements de coût de quelques grands projets au Canada, au Québec et ailleurs dans le monde

\begin{tabular}{|c|c|}
\hline $\begin{array}{l}\text { Dépassement de coût de grands projets au Canada } \\
\text { et au Québec }\end{array}$ & $\begin{array}{l}\text { Dépassement de coût de grands projets dans le } \\
\text { reste du monde }\end{array}$ \\
\hline $\begin{array}{l}\text { Stade Olympique de Montréal } \\
\left(134 \text { millions } \$^{1} ; 264 \text { millions } \$^{2}\right)\end{array}$ & Aéroport international de Denver : $200 \%$ \\
\hline $\begin{array}{l}\text { Registre des armes à feu du Canada } \\
(119 \text { millions \$; plus de } 1 \text { milliard \$) }\end{array}$ & $\begin{array}{l}\text { Boston Big Dig } \\
(2,6 \text { milliards } \$ ; 14,6 \text { milliards } \$)\end{array}$ \\
\hline $\begin{array}{l}\text { Centre Rogers (auparavant, Toronto Sky Dome) } \\
(225 \text { millions \$; environ } 600 \text { millions \$) }\end{array}$ & Parlement écossais : $4300 \%$ \\
\hline $\begin{array}{l}\text { The Syncrude Upgrader Expansion } 1 \text { Project, } 2006 \\
(4,1 \text { milliards } \$ ; 7,8 \text { milliards) }\end{array}$ & $\begin{array}{l}\text { Pont San Francisco-Oakland } \\
(1,3 \text { milliard } \$ ; 6,4 \text { milliards } \$)\end{array}$ \\
\hline $\begin{array}{l}\text { Musée des civilisations du Canada } \\
\text { (80 millions \$; } 340 \text { millions \$) }\end{array}$ & $\begin{array}{l}\text { Satellite-espion du Pentagone: } \text { dépassement : } \\
4 \text { milliards }\end{array}$ \\
\hline $\begin{array}{l}\text { Rapibus, Gatineau } \\
\text { (150 millions } \$ ; 300 \text { millions } \$ \text { ) }\end{array}$ & Station spatiale : dépassement 5 milliards \\
\hline $\begin{array}{l}\text { Phase 1 CNRL Horizon Project, } 2009 \\
\text { (6,8 milliards } \$ ; 9,7 \text { milliards) }\end{array}$ & $\begin{array}{l}\text { Jeux Olympiques de Sotchi } \\
(10 \text { milliards } \$ ; 50 \text { milliards } \$)\end{array}$ \\
\hline
\end{tabular}




\section{GRANDS PROJETS, GRANDS PROBLÈMES ET GRANDES DÉSILLUSIONS?}

On l'a vu, la performance de coût des grands projets laisse à désirer. Mais c'est la performance tout entière des grands projets qui déçoit bien des parties prenantes. Et ce n'est pas des exemples qui manquent. Prenez le projet de transport en commun, le Rapibus, le Système rapide par bus peut-être le plus grand projet dans l'histoire moderne de la ville de Gatineau. Le Rapibus, financé à $75 \%$ par Québec et à $25 \%$ par la ville de Gatineau, offre un corridor exclusif aux autobus sur plus de $15 \mathrm{~km}$ entre le secteur Gatineau et les centres-villes du secteur Hull et de la ville voisine d'Ottawa. Livré le 19 octobre 2013 avec trois (3) ans de retard sur l'échéancier initial et un dépassement de budget de près de $100 \%$ pour un coût total réel d'environ 300 millions de dollars, malgré l'abandon du tronçon entre les boulevards Labrosse et Lorrain, il a fait l'objet de fortes critiques dans la presse et continue de susciter beaucoup de controverses. Pourtant d'autres villes avant Gatineau, comme Ottawa et Pittsburgh, ont réalisé leur Rapibus à elles!

En effet, à peine mis en service, le Rapibus sensé être un système rapide et efficace de transport en commun, à l'échelle de la région de l'Outaouais, semble avoir pris des allures de «Lentibus »! C'est $\mathrm{du}$ moins le surnom que lui ont donné ses détracteurs - malheureusement des bénéficiaires mécontents du service - qui raillent la lenteur du service et son inefficacité car, pour eux, leur temps de transport a dû augmenter de quelques dizaines de minutes avec le Rapibus. Même s'il aura pris 17 ans $(1992$ - 2009) pour le planifier et quatre autres pour le réaliser (novembre 2009 - octobre 2013), rien n'y fit. Peu de temps après sa mise en service à l'automne 2013, des pétitions en ligne, des plaintes et des manifestations en pleine campagne électorale municipale sans oublier des commen-taires peu élogieux dans les réseaux sociaux comme Facebook et Twitter l'ont accueilli. Le Rapibus a, semble-t-il, coûté son poste au maire sortant, Marc Bureau. Le nouveau maire, Maxime Pedneau-Jobin, qui a promis de régler le problème pendant la campagne électorale, a, pour ce faire, pris la présidence de la Société des Transports de l'Outaouais (STO). Malgré près d'un million et demi de dollars débloqués pour effectuer des ajustements conséquents, dont l'ajout de lignes Express - initialement délaissées par le Rapibus - des centaines d'usagers boudent et désertent le transport en commun...

\section{Les grands projets posent de grands défis de gestion, occasionnent de grandes difficultés récurrentes et deviennent parfois, voire souvent des histoires d'horreur. Et pour cause, ils sont complexes et entourés d'incertitude.}

Comme le Rapibus, les grands projets posent de grands défis de gestion, occasionnent de grandes difficultés récurrentes et deviennent parfois, voire souvent des histoires d'horreur. Et pour cause, ils sont complexes et entourés d'incertitude. Ils se caractérisent par des temps de planification et de réalisation longs, des interfaces complexes, des parties prenantes aux intérêts divergents, voire conflictuels, un grand attrait pour les politiciens, une visibilité remarquable, une présence médiatique forte et une envergure et une ambition qui changent avec le temps.

Par ailleurs, les grands projets s'exposent au biais de l'optimisme - même les experts ont tendance à en sous-estimer les coûts et à croire qu'ils risquent moins que quiconque de se tromper sur leurs prévisions - ou à l'illusion de la planification (planning fallacy, voir les travaux du prix Nobel d'économie Kahneman ${ }^{16}$ ) ou l'art de présenter des plans de projet qui se rapprochent trop des estimations optimistes et que seules des statistiques des projets similaires, mais passés peuvent améliorer. Le mensonge stratégique, c'est-à-dire, le stratagème qui consiste à sous-estimer les coûts du projet et à en surestimer les avantages en vue de le faire accepter et d'obtenir du financement ô combien nécessaire afin de le réaliser n'est pas non plus rare dans les grands projets. Ainsi, le choix porte souvent, non pas sur les «bons » projets, mais plutôt sur ceux qui paraissent bien sur papier $^{17}$. Comment peut-on comprendre que les jeux Olympiques d'hiver de Sotchi aient été estimés à 10 milliards $\$$ sachant que ceux de Londres auront coûté officiellement plus de 18 milliards \$ (alors que l'on les estimait à environ 
3 milliards \$!) si ce n'est que par l'effet combiné $\mathrm{du}$ biais de l'optimisme et $\mathrm{du}$ mensonge stratégique?

Sans doute, les problèmes de gestion auxquels les grands projets font face sont légion. On peut noter des critères de succès flous, un changement de stratégie du champion du projet (sponsor), une piètre conception, planification ou réalisation, une incertitude technique et de conception, des conditions géophysiques peu propices, un avantprojet bâclé, une mauvaise estimation des délais et des coûts, une assurance qualité défaillante, une gestion des contrats et des approvisionnements hasardeuse, un manque d'expérience, une rotation excessive $\mathrm{du}$ personnel, des problèmes de financement ou d'inflation et une mauvaise gestion des risques et du changement en cours de réalisation, etc. ${ }^{18}$

$\mathrm{Au}$ total, les grands projets connaissent des problèmes contextuels, institutionnels, et managériaux comme c'est le cas dans le domaine du développement international ${ }^{19}$. Prenez encore le cas du Rapibus. D'abord des problèmes de gestion: un contrôle de projet et un suivi défaillants et peu transparents, des problèmes informatiques avec l'outil Plani-Bus, qui permet aux futurs usagers de consulter les horaires et les nouveaux trajets du Rapibus et de planifier conséquemment leurs déplacements, un manque de stationnements incitatifs dans le secteur Gatineau de la ville. Peut-être parce que, comme bien des politiciens, il ne maitriserait pas la gestion de projet, le maire sortant, Marc Bureau, n'a pas défendu ou n'a pas su défendre son projet!

Ensuite des problèmes contextuels. Le Rapibus a dû faire face à une présence plus importante de sols contaminés sur son tracé qui a accueilli des trains pendant de nombreuses années. Des ouvrages d'art le long du corridor ont dû subir un ajustement pour tenir compte des profils et des conditions géotechniques des terrains. Ainsi la réserve budgétaire de 49,5 millions $\$$ planifiée pour faire face aux imprévus n'a point suffi.

\section{Le mensonge stratégique, c'est-à-dire, le stratagème qui consiste à sous-estimer les coûts du projet et à en surestimer les avantages en vue de le faire accepter et d'obtenir du financement ô combien nécessaire afin de le réaliser n'est pas non plus rare dans les grands projets.}

Enfin, sur le plan institutionnel, le Vérificateur Général de la ville de Gatineau a reproché à la STO de ne pas avoir signé un protocole d'entente avec le bailleur de fonds du projet: le ministère des Transports du Québec. Une simple lettre de garantie signée de la main du ministre serait insuffisante. Mais y a-t-il des solutions aux problèmes des grands projets?

\section{GRANDS PROJETS : TROP GRANDS POUR RÉUSSIR?}

Le triangle délai, coût, qualité représente le succès de la gestion et se mesure souvent dès la fin de la réalisation du projet alors que le succès du livrable renseigne sur l'utilité, la valeur et la contribution des résultats du projet et se mesure souvent bien après la livraison du projet au client.

Le succès des projets est le sujet de prédilection des chercheurs et des praticiens de la gestion de projet. Le moteur de recherche Google, en date du 20 février 2014, affiche 22700000 résultats pour l'énoncé de recherche «succès des projets » et 385000000 pour l'énoncé «project success »! Mais si les uns et les autres ne s'entendent pas complètement sur la définition ni la mesure du succès des projets, ils s'accordent sur deux dimensions du succès des projets : le succès de la gestion (donc du processus) et le succès du livrable (donc du produit ou service qui découle du projet). On convient aussi que le moment de la mesure est important dans l'évaluation du projet et de ses résultats. Ainsi, le triangle délai, coût, qualité représente le succès de la gestion et se mesure souvent dès la fin de la réalisation du projet alors que le succès du livrable renseigne sur l'utilité, la valeur et la contribution des résultats du projet et se mesure souvent bien après la livraison du projet au client. À l'aune de cette double dimension du succès qui se décline dans le temps, les grands projets offrent un mélange de réussite et d'échec 
dans le temps. Quelques exemples pour s'en convaincre.

D'abord des fiascos complets ou plutôt des échecs retentissants tant de la gestion que du livrable; ou encore des échecs à la fois à court terme par rapport au processus de gestion et, à moyen et long termes par rapport aux résultats du projet. C'est le cas des éléphants blancs. L'aéroport de Mirabel offre une belle illustration. Le Concorde, par exemple, a connu des dépassements de délai et de coût, mais également un fiasco commercial. Il en est ainsi du Registre des armes à feu du Canada qui est passé d'un budget de 114 millions de dollars à un coût réel dépassant le milliard de dollars avant d'être tout simplement abandonné, par les conservateurs fédéraux, pour des raisons politiques.

Ensuite des échecs de gestion, mais de grands succès pour le livrable. Par exemple, le Musée canadien des civilisations (désormais Musée canadien de l'histoire) budgété pour 80 millions \$ a finalement coûté 340 millions \$, mais est l'une des destinations touristiques importantes du Canada. Si le fabricant automobile Ford a congédié le gestionnaire du projet de réalisation de la première génération de Ford Taurus (1986) pour les trois mois de retard que le projet a connus, cette voiture a connu un succès commercial retentissant pour Ford, quelques années plus tard (plus de 2 millions de voitures vendues!).

Il y a des grands projets qui connaissent un succès de gestion, mais un fiasco du livrable. Le gestionnaire de la troisième génération de Ford Taurus (1995), tirant les leçons des déboires de son prédécesseur, a livré le projet dans les délais, mais cette voiture a été un fiasco commercial pour Ford. Le projet du pipeline TchadCameroun réalisé par ExxonMobil (1993- 2003; 3,7 milliards \$) a été livré dans les délais et considéré par la Banque mondiale comme un modèle de gestion des projets d'infrastructure en Afrique; mais il n'a pas pu livrer l'impact attendu en matière de développement.

Enfin, il y a des projets qui sont considérés comme des grands succès tant en ce qui concerne le processus de leur gestion que leurs résultats. Pensez au projet Apollo de la NASA (1966-1972) qui a été livré dans les délais et qui a été un grand succès stratégique pour les États-Unis. Les grands projets semblent alors un mélange de réussite et d'échec dans le temps.

Le temps n'est pas à négliger dans leur évaluation. Les grands projets requièrent parfois de la patience et du bricolage ou une sorte d'essai-erreur. C'est le cas du projet du fameux système d'exploitation Windows. De l'avis même de Steve Ballmer, cofondateur de Microsoft, Windows 1.0 n'a pas été un succès; Windows 2.0 non plus; mais Windows 3.0 a été un succès retentissant!

De plus, l'échec de certains grands projets amène au succès des autres. Voici quelques exemples probants. Le projet de Christophe Colombe d'atteindre la route des Indes a raté non seulement sa cible, mais également le temps et le budget planifiés. Toutefois, la découverte plutôt fortuite du Nouveau Monde est un grand succès! Aussi, comme le souligne Bill Gates, les logiciels à succès de la suite Microsoft tels qu'Excel et Access tirent respectivement leurs succès du fiasco lamentable d'un tableur qui n'a pu tenir devant le concurrent Lotus 1-2-3 et d'une base de données appelée OMEGA qui a coûté, en vain, des millions \$.

Mais s'il semble que les grands projets dépassent souvent les délais et les budgets, qu'ils représentent un mélange de succès et de réussite dans le temps, peut-on pour autant avancer que la taille, notamment le budget des grands projets est un enjeu crucial pour leur succès? Peu d'études ont abordé le lien statistique entre la taille et la performance des projets. Mais que l'on définisse la taille par la durée, le budget ou l'envergure (ex. : le nombre de membres dans l'équipe de projet, le nombre d'heures-personnes), ces études ne semblent pas concluantes. De l'avis de plusieurs experts, dont Flyvbjerg, ce n'est pas la taille, l'envergure ou le budget des grands projets qui les rend complexes, mais plutôt leur durée. En effet, il semble ne pas y avoir de lien statistiquement significatif entre le budget et la performance des projets, notamment dans le secteur des $\mathrm{TI}^{20}$. Mais même si Reich et al. ${ }^{21}$ avancent que la taille des projets de TI ne semble pas être un enjeu crucial dans leur succès, ils suggèrent qu'au-delà de 40 millions \$, le risque d'échec des projets est plus grand. Le Groupe Standish penche clairement en faveur des petits projets de TI. 
Quoi qu'il en soit, les statistiques de performance laissent penser que les grands projets semblent trop grands pour réussir dans les délais et les coûts. Mais leurs promoteurs ne les réalisent pas seulement pour leur plaisir: comme le suggère Woody Allen, ils le font pour leurs « œufs »! Oui, les grands projets regorgent d'avantages qui vont au-delà des actifs physiques et surtout d'un potentiel d'impact transformationnel, sur les plans économique, social et humain, irrésistible, et c'est

\section{CONCLUSION}

\section{Les grands projets ne sont pas trop grands} pour réussir et, ainsi, livrer l'impact attendu; ils sont d'abord trop grands pour échouer, en cours de réalisation, et ainsi faire l'objet d'abandon; et ils sont surtout trop grands pour réussir dans les délais et les coûts.

Grands projets, grandes réalisations. «Big is beautiful». Voilà qui explique le phénomène du «too big to fail» au niveau des projets. Souvent dans un élan plutôt sur-optimiste et dans une sorte d'illusion de la planification, comme le souligne Flyvbjerg, ou plutôt par simple ignorance, comme le prétend Hirschman avec son principe de la « main cachée » (Hiding Hand), les promoteurs des grands projets en sous-estiment les coûts et en surestiment les avantages. Souvent, ils font valoir qu'il est tard voire trop tard d'y renoncer, en cours de réalisation, compte tenu des précieux avantages potentiels des grands projets et des coûts irrécupérables, mais importants jusque-là investis. Ainsi, les grands projets semblent souvent trop grands pour échouer... en cours de réalisation. $\mathrm{Ne}$ pas les abandonner, c'est souvent accepter des dépassements élevés de délai et de coût.

Toutefois, malgré des dépassements de coût réels, récurrents et généralisés, il ne semble pas y avoir de lien statistique entre le budget et la performance des projets. Oui, aucun projet n'est ni trop grand ni trop petit pour échouer par rapport à ses objectifs et aucun secteur d'activité n'y échappe. Mais, grands projets, grands défis. Grande complexité. Grande incertitude. Grandes controverses. Et des risques de grandes désillusions en cas d'échec. Les grands projets offrent un mélange de réussite et ce qui fait leur charme. C'est pourquoi les grands projets paraissent également souvent trop grands pour échouer en cours de réalisation, c'est-à-dire, que leurs avantages souvent grandioses paraissent trop grands à leurs promoteurs pour qu'ils y renoncent malgré des dépassements de délai et de coût exorbitants. Souvent ils semblent trop grands pour qu'on les abandonne. Et en fin de compte, quand ils échouent, les désillusions sont aussi trop grandes et la critique virulente.

d'échec dans le temps et l'échec des uns peut même amener à la réussite des autres.

En somme, les grands projets ne sont pas trop grands pour réussir et, ainsi, livrer l'impact attendu; ils sont d'abord trop grands pour échouer, en cours de réalisation, et ainsi faire l'objet d'abandon; et ils sont surtout trop grands pour réussir dans les délais et les coûts. À l'annonce du prochain grand projet autour de vous, méfiez-vous de sa réserve budgétaire car, aussi grande soit-elle, elle va disparaître comme une étoile filante! Peutêtre retiendrez-vous le conseil de Nevan Wright Jr. : bonifiez de 50\% l'estimation de coût de chaque activité du projet et ajoutez $50 \%$ à la première estimation globale du coût total du projet!

\section{BIBLIOGRAPHIE ET NOTES}

${ }^{1}$ Lavagnon IKA, PhD, est professeur de gestion de projet à l'École de gestion Telfer de l'Université d'Ottawa. Email : Ika@telfer.uottawa.ca.

${ }^{2}$ KPMG (2013). « Megaprojects », Insight - The Global Infrastructure Magazine, 4, 1-74.

${ }^{3}$ Voir pour le cas des projets de TI, Eveleens, J. L. et Verhoef, C. (2010). «The rise and fall of the Chaos report figures ». IEEE Software, Janvier/Février, 30-36. ${ }^{4}$ Flyvbjerg, B. (2005). «Policy and planning for large infrastructure projects: Problems, causes, cures». World Bank Policy Research Working Paper 3781.

${ }^{5}$ Hirschman, A. O. (1967). Development Projects Observed. Washington, DC : Brookings Institution.

${ }^{6}$ Merrow, E., McDonnell, I. L.et Argüden, R.Y. (1988). Understanding the outcomes of megaprojects: a quantitative analysis of very large civilian projects, Rand Corporation Publication Series \#R-3560-PSSP, March.

${ }^{7}$ Morris, P. W. G. et Hough, G. H. (1987). The Anatomy of major projects, John Wiley and Sons : New York. 
${ }^{8}$ Miller, R. et Lessard, D. R. (2000). The Strategic management of large engineering projects, MIT Press : Cambridge.

${ }^{9}$ Meier, S. R. (2008). « Best project management and systems engineering practices in pre-acquisition practices in the federal intelligence and defense agencies » Project Management Journal, 39, 1, 59-71.

${ }^{10}$ Voir Flybjerg, 2005, op. cit.

${ }^{11}$ Hertogh, M., Baker, S., Staal-Ong, P.L. et Westerveld, E. (2008). Managing large infrastructure projects Research on best practices and lessons learnt in large infrastructure projects in Europe, Netlipse.

${ }^{12}$ OMEGA (2012). Mega projects Executive Summary. Lessons for decision-makers: An analysis of selected large-scale transport infrastructure projects. Omega Centre, Décembre.

${ }^{13}$ THE STANDISH GROUP (2013). Chaos Manifesto 2013. Think big, act small. The Standish Group.

${ }^{14}$ Flyvjberg, B. et Budzier, A. (2011). «Why your IT project may be riskier than you think ». Harvard Business Review, Septembre, 23 - 25.

${ }^{15}$ Jergeas, G. F. et Ruwanpura J. (2010). Why cost and schedule overruns on mega oil sands projects? ASCE Practice Periodical on Structural Design and Construction 15(1) : 40-43.

${ }^{16}$ Kahneman, D. (2011). Thinking fast and slow. DoubleDay, Canada.

${ }^{17}$ Voir Flyvbjerg, 2005, op. cit.

${ }^{18}$ Voir Morris et Hough (1987), op. cit.

${ }^{19} \mathrm{Ika}$, L.A. (2012). «Project management for development in Africa: Why projects are failing and what can be done about it ", Project Management Journal, 43(4), 27-41.

${ }^{20}$ Voir Flybvjerg et Budzier 2011, op. cit.

${ }^{21}$ Sauer, C., Gemino, A. et Reich, B.H. (2007). "Managing projects for success: The impact of size and volatility on IT project performance ", Communications of the ACM, 50(11), 79-84. 


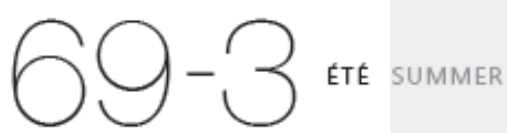

\section{REVUE TRIMESTRIELLE}

RELATIONS INDUSTRIELLES

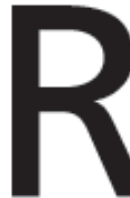

Revue trimestrielle bilingue publiée depuis 1945 par le Département des relations industrielles de l'Université Laval

ARTICLES

Conflits travail-famille et intention de quitter dans le secteur de la santé

AZE RHNIMA, THIERRY WILS, CLAUDIO E. POUSA ET MELANIE FRIGON

Organizational Contexts for Union Renewal KYOUNG-HEE YU

Étude des conflits inter-rôles: les hommes gestionnaires ou

professionnels en fonction de leur profil d'engagement dans les rôles de vie ERIC JEAN ET LISE LACHANCE

Dialing it in: A Missed Opportunity regarding the Strategic Use of Telework? GORDON B. COOKE, JAMES CHOWHAN AND TOM COOPER

Le prêt de main-d'œuvre : un dispositif innovant au service des territoires

LUDMNE CALAMEL ET VIRGINIE GALLEGO-ROQUELAURE

The Relationship between the Internal Labour Market and Transitions from Temporary to Permanent Employment in Korea HYONDONG KIM AND DONG-JIN LEE

Envergure du poste et engagement :

le rôle modérateur du soutien et des relations avec le supérieur

CATHERINE MARCHAND ET CHRISTIAN VANDENBERGHE

\section{INDUSTRIAL RELATIONS \\ QUARTERLY REVIEW}

A bilingual quarterly published since 1945 by the Industrial Relations Department, Université Laval

RI/IR EN LIGNE

RVIR est disponible en ligne sur le site Erudit : www.erudit.org/revue/ri

Pour abonnement à la version numérique, contacter Érudit.

Pour consulter les sommaires des numéros publiés, les résumés d'articles ou pour vous abonner à la version papier, visitez notre site Internet :

www.riir.ulaval.ca

\section{RI/IR ONLINE}

$\mathrm{RVIR}$ is available on line

on Erudit website at:

www.erudit.org/revue/ri

To subscribe to the online version, please contact Erudit.

Visit our website for contents listings, abstracts, or to subscribe to the print edition:

www.riir.ulaval.ca

RELATIONS INDUSTRIELLES INDUSTRIAL RELATIONS

Pavillon J.-A.-DeSève

1025 , avenue des Sciences-Humaines

Bureau 3129, Université Laval

Québec (Québec) Canada G1V 0A6

TÉLÉPHONE : (418) 656-2468

COURRIEL / E-MAIL :

relat.ind@rlt.ulaval.ca

www.riir.ulaval.ca 